\title{
Introduction (version française)
}

Introduction

\section{Florence Labaune-Demeule}

\section{(2) OpenEdition}

Journals

Édition électronique

URL : http://journals.openedition.org/transtexts/1397

DOI : $10.4000 /$ transtexts. 1397

ISSN : 2105-2549

Éditeur

Gregory B. Lee

Référence électronique

Florence Labaune-Demeule, «Introduction (version française) 》, Transtext(e)s Transcultures 跨文本跨文 化 [En ligne], 15 | 2020, mis en ligne le 14 décembre 2020, consulté le 29 janvier 2021. URL : http:// journals.openedition.org/transtexts/1397; DOI : https://doi.org/10.4000/transtexts.1397

Ce document a été généré automatiquement le 29 janvier 2021.

(c) Tous droits réservés 


\title{
Introduction (version française)
}

\author{
Introduction
}

Florence Labaune-Demeule

1 Dans l'entrée «Censure » de l'Encyclopédie Universalis, Julien Duval explique toute la complexité de ce terme puisque ce dernier permet de désigner à la fois «l'action de condamner un texte ou une opinion, d'en interdire sa diffusion, et l'institution qui prononce cette condamnation. $»^{1} \mathrm{Il}$ ajoute que ce terme «trouve son origine dans une institution de la République romaine, celle des censeurs, deux magistrats chargés tous les cinq ans d'évaluer (en latin censere) le nombre des citoyens, de les répartir en classes en fonction de leur richesse, et d'exclure de ces listes les citoyens de 'mauvaises mœurs' ». La censure équivaut alors à déplacer, "déclasser » des citoyens romains selon des critères patrimoniaux et moraux. À partir du Moyen-Âge, le terme censure en était venu à renvoyer à « une institution officielle qui, pour toute publication d'un écrit ou représentation d'un spectacle, délivre une autorisation préalable et peut exiger pour cela des modifications ou des suppressions de passages de l'œuvre. " D'une fonction «comptable » et moralisatrice, on était donc passé à une institution s'octroyant un droit de regard et une autorité morale et culturelle permettant de protéger les valeurs sociétales à transmettre. Au lieu d'inscrire les membres de la société sur ses tablettes comme à l'époque romaine, elle avait pour objet d'effacer, de rendre inaccessible au regard ou à la connaissance ce qui n'était pas jugé conforme. Le déplacement du citoyen hors des catégories sociales requises était donc renforcé par l'effacement de son discours.

2 Julien Duval remarque par ailleurs que, bien que de telles formes de censure radicale aient été moins souvent actives en Europe depuis le dix-neuvième siècle sous l'influence des révolutions française et américaine notamment et sous celle des questions relatives aux Droits de l'Homme, les manifestations contemporaines de la censure sont toujours bien présentes, en particulier dans les pays gouvernés par des pouvoirs autoritaires. Selon qu'il est utilisé dans son sens général ou dans un sens plus métaphorique, le terme «censure » peut renvoyer aux censeurs mais aussi aux rôles et fonctions d'institutions (qu'il s'agisse de censure religieuse, politique, étatique, économique ou autre) ou aux différents moyens répressifs mis en œuvre pour dissuader 
quiconque d'adopter des positions « condamnables ». ${ }^{2}$ Lélan moralisateur qui motive les actes des censeurs peut ainsi s'appuyer sur un désir de réforme, sur une volonté de ramener dans le droit chemin, la censure étant, dans ce cas, synonyme de punition, que ce soit en imposant une autorité de manière silencieuse et insidieuse ou en usant de la violence et de l'emprisonnement comme moyens coercitifs pour, d'une certaine manière, "ré-éduquer " l'individu, le conduire à adhérer à la norme sociale requise. Cependant, comme l'explique André Lalande dans son Vocabulaire technique et critique de la philosophie, la censure peut aussi se définir comme un acte psycho-analytique, Freud utilisant ce terme pour renvoyer à la fonction psychique qui empêche, voire interdit, les manifestations spontanées et sincères de désirs et images mentales réprimées, qui s'expriment à travers carences, travestissements ou transformations symboliques des faits conscients correspondants. ${ }^{3}$

Dans le cadre des productions postcoloniales, la position de Frantz Fanon n'est pas très éloignée de cette signification lorsqu'il explique que, à l'époque coloniale et postcoloniale, les Noirs étaient perçus - à la fois de manière auto-réflexive et réciproque - comme objets plutôt que comme sujets, que leurs identités ont été déplacées de telle sorte qu'ils ne pouvaient pas se voir tels qu'ils étaient mais tels qu'ils auraient dû être selon les valeurs coloniales. Pour faire écho à la théorie de Frantz Fanon, John McLeod commente les images d'amputation et l'utilisation du champ sémantique militaire souvent associé à ce genre de censure par Fanon :

The violence of this 'revision' of his identity is conveyed powerfully in the image of amputation. Fanon feels abbreviated, violated, imprisoned by a way of seeing that denies him the right to define his own identity as a subject. [...] That imaginative distinction that differentiates between 'man' (self) with 'black man' (other) is an important, devastating part of the armoury of colonial domination, one that imprisons the mind as securely as chains imprison the body... ${ }^{4}$

De telles métaphores, empruntées au domaine médical et militaire, permettent de proposer des représentations visuelles de la censure pour exprimer à la fois l'absence de liberté et la domination d'une part, et l'impossible représentation de soi induite par la censure d'autre part. Aux formes plus ouvertes ou explicites de censure institutionnelle s'ajoutent donc des moyens plus insidieux ou implicites, prenant parfois la forme d'autocensure. L'individu s'impose alors des limites à lui-même, qui l'empêchent d'exprimer ce qu'il pense, ou même d'oser penser à certains éléments. Dans de tels cas, la censure envahit la vie quotidienne de façon insidieuse, notamment dans des sociétés qui y furent autrefois - et parfois longuement - soumises. Cette idée a également été abordée par Pierre Bourdieu (parmi d'autres penseurs), qui a identifié les processus sociologiques à l'œuvre dans tout acte de communication. En effet, Bourdieu a insisté sur le fait que la censure renvoyait à la fois à des formes institutionnelles de contrôle et à toute forme de discours individuel qui, à son tour, représente les transactions ou compromis entre ce que le locuteur a l'intention de dire à son interlocuteur et ce que l'interlocuteur lui-même est prêt à entendre, ou ce qu'il peut simplement comprendre. En effet, pour Bourdieu, le schéma communicationnel est basé sur la légitimité et sur la nécessité ressentie par les individus de contrôler leur discours (d'où de fréquentes ou constantes situations d'autocensure), afin de répondre aux règles fixées par le groupe social auquel appartient l'individu ou pour répondre aux règles du champ dans lequel évolue ce dernier. ${ }^{5}$ L'interaction entre les règles intégrées par cette personne et celles qui définissent le champ affecte le processus de 
communication. Il en résulte une forme inconsciente de censure dans le discours du locuteur afin de limiter toute déviance importante des règles établies. ${ }^{6}$

5 Si la censure ne s'est pas toujours manifestée de manière explicite par l'interdiction de publication de certains textes pendant l'ère impériale ou coloniale, elle n'en a toutefois pas moins conduit à adopter des positions ou stratégies insidieuses, à imposer le silence, à nier le discours de l'autre sous de multiples formes, voire à favoriser une certaine autocensure. Ania Loomba soulève cette question de manière explicite dans Colonialism/Postcolonialism lorsqu'elle se demande «[d]ans quelle mesure le pouvoir colonial est-il parvenu à réduire le colonisé au silence? ".7 Bill Ashcroft, Gareth Griffiths et Helen Tiffin attirent aussi l'attention sur ce point lorsqu'ils évoquent l'impact du colonialisme sur les populations colonisées lorsqu'ils étudient le « centre impérial qui a réduit au silence et marginalisé la voix postcoloniale, l'abrogation de ce centre impérial au sein du texte et l'appropriation active de la langue et de la culture de ce même centre $\aleph^{8}$ Ces théoriciens montrent en effet que la censure institutionnalise le silence en "muselant la voix des individus ». ${ }^{9}$ Prenant l'exemple de la censure sudafricaine, ils écrivent :

This silence is literally and dramatically revealed in the censorship exercised by the government over newspapers, journals, and much creative writing. It has two aspects: there is the literal silencing which will not permit the freedom necessary to appropriate language, and there is the further silence which necessarily precedes the act of appropriation. ${ }^{10}$

6 En fait, l'utilisation du discours, donc l'actualisation de la langue, est un facteur essentiel. En effet, les colonisés, d'abord réduits au silence, sont aussi confrontés à la domination dans leur usage de la langue : pour la plupart des écrivains postcoloniaux, cette dernière reste celle « $\mathrm{du}$ colonisateur»; elle est un marqueur de leur appartenance à la périphérie coloniale puisqu'elle ne permet pas de représenter et de signifier d'emblée la vérité de l'expérience vécue dans un lieu où une autre langue serait plus adéquate. Il leur faudra se ressaisir de la langue dominante et lui donner un nouveau sens « décentré » pour inscrire leur existence dans le monde (et leur vision du monde) par un discours devenu audible et signifiant :

If language constructs the world then the margins are the centre and may reconstruct it according to a different pattern of conventions, expectations, and experiences. ${ }^{11}$

7 Les littératures postcoloniales ont toujours eu pour objet de s'affranchir de contraintes, notamment celles imposées par l'impérialisme, de s'opposer aux idées du colonialisme et de combattre ce dernier par un travail de sape des clichés coloniaux. Certains concepts entérinent en effet la domination coloniale, comme (pour n'en mentionner que quelques-uns), celui d'un centre impérial et de colonies périphériques, ou celui de la mission civilisatrice de la Grande-Bretagne et d'autres pays européens, ou encore celui de l'image construite par l'Orientalisme et dénoncé par Said. ${ }^{12}$

8 Elleke Boehmer donne une définition intéressante du rôle de la littérature postcoloniale :

Rather than simply being the writing which 'came after' empire, postcolonial literature is that which critically scrutinizes the colonial relationship. It is writing that sets out in one way or another to resist colonialist perspectives. As well as a change in power, decolonization demanded symbolic overhaul, a reshaping of dominant meanings. Postcolonial literature formed part of this process of overhaul. ${ }^{13}$ 
La littérature postcoloniale est donc décrite à la fois comme une littérature de résistance et d'opposition, et comme une littérature de révision et de refondation en ce qu'elle modifie la perspective dominante.

10 On mesure ainsi qu'une domination s'établit certes par ce que Justin D. Edwards appelle «le pouvoir du fusil» mais aussi par ce qu'il nomme «le pouvoir du discours », qu'il définit comme « la manipulation de la langue et de la pensée [qui] devient une forme de contrôle qui accroît la puissance du colonisateur et assujettit le natif. $»^{14}$

11 Étudier les mécanismes à l'œuvre lorsque l'on veut taire quelque chose ou faire taire quelqu'un est donc nécessaire pour dévoiler la domination et l'infériorisation que les «Subaltern Studies » ont mises en avant suivant la théorie de la subalternité de Ranajit Guha et les arguments développés par Gayatri Chakravorty Spivak dans son essai « Can the Subaltern Speak? ». La maitrise du discours, voire le contrôle de ce dernier, sont toujours au cœur des stratégies de censure et, selon Spivak, ils témoignent d'une forme de violence épistémique par l'action même de réduire au silence. Justin D. Edwards explique aussi clairement que l'absence de toute reconnaissance d'un acte de langage par le destinataire d'un message vise à nier l'existence même de celui qui le formule. Il ne s'agit pas seulement d'un refus de communication, mais de la négation de l'existence même de l'autre :

Epistemic violence arises when those people who hold power begin to silence a less powerful group. It thus points to the interplay between the pre-colonial and colonial structures of dominant discourses that erase the space from which the subaltern can speak. [...] Often, the subaltern makes an attempt at selfrepresentation, yet this act of representation is not heard. The hegemonic listener does not recognize it, because it does not fit into the official institutional structures of representation. [...] [W]ithin the very definition of subalternity there is an implicit not-being-able-to-make-speech-acts, for if the subaltern could speak she would no longer be a subaltern. ${ }^{15}$

Inversement, il en découle que la prise de parole et la reconnaissance de la voix de l'autre attestent de son existence et de son identité. C'est donc en acceptant d'entendre le discours postcolonial que la voix et l'identité des périphéries peuvent être établies.

Lorenzo Mari s'interroge alors sur la raison qui expliquerait qu'aucune définition de la "censure postcoloniale» n'ait été avancée et il observe que si l'allégorie est «le principal mode rhétorique » de la littérature soumise à la censure, l'usage qui en est fait dans un contexte postcolonial a un impact plus spécifique : ${ }^{16}$

The nexus of allegory and history turns out to be different in Western literature, where allegories are concerned either 'with redeeming or recuperating the past', and in postcolonial literature, where allegories specifically intend to transform 'the imperial myth of history' (1988: 158). By restoring those histories which were systematically denied and/or omitted within colonial narratives, postcolonial literature struggles against the silence imposed on the colonized populations as a peculiar form of colonial censorship (Chin 2009). At the same time, the transformation of history which is inherent to postcolonial literature is based on a series of elements which are inevitably marked as 'new' and 'other'. This leads to a case for 'paradoxical doubleness or ambivalence', as postcolonial literature is 'already constituted within institutional and generic constraints whose work it is to package and displace the counter-discursive force [...] under a sign of secondariness, derivation, simulacrum, or mimicry' (Slemon 1989: 100). ${ }^{17}$ 


\section{cherche ainsi à combattre le silence imposé, que ce soit par la domination et} l'infériorisation établies par le colonialisme ou par toute autre forme de censure.

Cependant, la littérature postcoloniale se doit aussi, parfois, de s'élever contre la censure institutionnelle de façon plus marquée, plus officielle, et c'est précisément le rôle de l'écrivain luttant pour sa liberté d'expression individuelle et collective de s'engager sur cette voie lorsqu'il y est contraint. Nul autre endroit ne fut plus soumis à la censure institutionnelle que l'Afrique du Sud pendant l'apartheid et la lecture des essais de Nadine Gordimer, par exemple, nous permet d'accéder aux motivations mais aussi au fonctionnement implicite de la lutte contre la censure étatisée.

Dans un essai publié en 1989 et intitulé «Censorship - The Final Solution. The Case of Salman Rushdie ", Nadine Gordimer décrit les manifestations excessivement violentes qui accompagnent souvent la décision prise par des gouvernements ou institutions d'interdire l'accès à certains livres. Selon elle, le paroxysme en fut atteint quelques mois après la publication des Versets sataniques de Salman Rushdie : ${ }^{18}$

Riots, book-burning, the demand that a work shall be banned worldwide, publishers boycotted, the threatened toppling of a prime minister, five dead - has ever a book been the pretext for such a frenzy of righteous barbarism? ${ }^{19}$

Si les émeutes, autodafés, interdictions, boycotts, exil et autres manifestations telles la fatwa qui frappa Rushdie, expriment des réactions violentes de la part des censeurs et parfois des citoyens, qui peuvent ainsi déchaîner leurs plus viles passions à l'encontre d'un contradicteur, Nadine Gordimer y perçoit «the unchanging principle of censorship, which was and is and always will be to harness the world to the tyrants' chariot. The tyrant may be a dictator, a regime, moral or religious bigotry $»{ }^{20} \mathrm{Il}$ s'agit donc toujours, à ses yeux, de limiter la liberté d'expression : la censure vise à taire ou à faire taire. ${ }^{21}$ Or l'écrivain, selon Nadine Gordimer, a la responsabilité de se manifester comme être social, c'est-à-dire comme membre d'une communauté ou d'une société propre. ${ }^{22} \mathrm{Il}$ est alors tiraillé entre deux pôles - celui de l'opprimé qui en fait souvent un porte-parole de sa souffrance, et celui de l'état, qui le punit parfois par la censure. ${ }^{23}$ Privilégier le premier pôle, par exemple par un activisme politique, conduit l'écrivain à subordonner sa créativité à des idées extérieures. Privilégier le second, c'est s'autocensurer. La véritable responsabilité d'un écrivain se trouve ailleurs : en suivant son intégrité créative, il lui faut exprimer ce qui lui paraît juste, ou vrai, que cela plaise ou non à la communauté dans laquelle il s'inscrit. ${ }^{24}$ Il lui faut avant tout adopter une position qui soit l'expression de son humanité : « Whether a writer is black or white, in South Africa the essential gesture by which he enters the brotherhood of man - which is the only definition of society that has any permanent validity - is a revolutionary gesture. $»^{25}$

Ce qui forme donc le geste essentiel de l'écrivain est sa faculté à transformer l'expérience grâce à sa propre créativité :

Writers who accept a professional responsibility in the transformation of society are always seeking ways of doing so that their societies could not ever imagine, let alone demand: asking of themselves means that will plunge like a drill to release the great primal spout of creativity, drench the censors, cleanse the statute books of their pornography of racist and sexist laws, hose down religious differences, extinguish napalm bombs and flame-throwers, wash away pollution from land, sea and air, and bring out human beings into the occasional summer fount of naked joy. [...] It could also be admitted that this is all writers can do: for creativity comes from 
within, it cannot be produced by will or dictate if it is not there, although it can be crushed by dictate. [...] The transformation of experience remains the writer's basic essential gesture; the lifting out of a limited category something that reveals its full meaning and significance only when the writer's imagination has expanded it. ${ }^{26}$ explique aussi que malgré l'élan créatif induit par la censure, cette dernière marque toujours les esprits des écrivains au fer de ses exigences. ${ }^{29}$ En effet, dès que les règles imposées par la censure furent assouplies en 1990 en Afrique du Sud, N. Gordimer prit davantage conscience des dangers que constituent les conséquences ou les soubresauts de la censure. Les écrivains devaient, selon elle, affronter leur imagination contrainte, voire distordue («cramped and even distorted imagination », Gordimer, p. 469), et devaient s'ouvrir individuellement à un nouveau "vocabulaire de vie " ( have to open themselves to a new vocabulary of life. ", Gordimer, p. 469), à des formes de créativité nouvelles, à de nouvelles stratégies d'écriture (ce qu'illustrent, par exemple le fréquent recours à l'allégorie, à l'allusion, ou au mode d'expression plus cryptique de la poésie, plutôt que l'adoption du genre plus explicite du roman réaliste, comme le firent de nombreux écrivains Noirs pendant l'Apartheid). Si l'empreinte que laisse la peur de la censure sur l'imagination de l'écrivain demeure à jamais, le rôle de ce dernier n'en reste pas moins frappé au sceau de l'engagement et de la responsabilité. Cette responsabilité devant l'humanité en général est aussi liée à l'ancrage de l'écrivain dans un contexte qui lui est propre, ce qui est particulièrement vrai pour les écrivains s'exprimant à l'ère postcoloniale. ${ }^{30}$

22 Ce recueil d'articles a donc pour ambition de proposer des approches variées de la censure dans un contexte postcolonial en adoptant une perspective transculturelle et transtextuelle : grâce aux différents textes et documents étudiés, écrits en français ou en anglais, le lecteur pourra se forger sa propre conception de ce qu'est la censure au prisme de la vision kaléidoscopique offerte par ce recueil. Pour ce faire, ce numéro de la revue Transtext(e)sTranscultures suit une articulation en deux parties. La première s'intéresse surtout à la manière dont les artistes postcoloniaux se positionnent et réagissent devant de multiples formes et manifestations de la censure, tandis que la seconde partie se concentre plus précisément sur les stratégies narratives à l'œuvre pour déjouer la censure.

23 Les multiples formes et manifestations de la censure ainsi que les moyens dont usent les artistes pour la dénoncer sont en effet ce qui rapproche les quatre premiers articles 
de ce recueil. Tout d'abord, l'essai de Charles Forsdick, «Between Censorship and Amnesia: The End of the Penal Colony in French Guiana» nous transporte dans la Guyane française coloniale et propose une étude de la colonie pénale et du bagne tristement célèbre qui furent établis à Cayenne. En analysant l'ouvrage de Léon Gontran Damas Retour de Guyane et celui d'Albert Londres Au Bagne parmi d'autres, C. Forsdick s'intéresse au milieu carcéral et nous offre une réflexion critique de l'institution pénitentiaire coloniale dont l'influence décroissante n'a fait qu'annoncer la grandeur et la décadence de l'empire colonial français. Il s'attache ensuite à proposer des visions plus contemporaines du bagne, par exemple en étudiant le photo-reportage de Chamoiseau et Hammadi, Guyane: Traces-mémoires du bagne, où les artistes essaient de percevoir ce que «les traces mémoires nous murmurent » afin de « défier la censure implicite de l'amnésie $» .{ }^{31}$

Le deuxième article nous conduit à marcher sur les pas de Doris Lessing en Rhodésie du Sud à l'époque coloniale. La contribution d'Hajer Elarem, qui s'intitule «Doris Lessing, the 'Prohibited Writer' Railing against Hegemonic Discourse", s'intéresse aux différents aspects de la censure dans le premier roman de Doris Lessing The Grass is Singing et montre comment la publication de ce premier ouvrage a conduit l'auteure à être censurée. Après avoir analysé dans quelle mesure le roman décrit la censure institutionnalisée, l'auteur de l'article se concentre sur le personnage de Mary Turner. Enfin, la troisième partie de l'article montre comment la publication de ce premier roman de $\mathrm{D}$. Lessing a conduit cette dernière à devoir être confrontée à la censure.

Comme l'indique ensuite le titre de l'article de Jacqueline Jondot «Censure et autocensure: les artistes de rue dans la 'révolution' égyptienne de Janvier 2011 », la censure se trouvait bien au centre de la situation à laquelle les artistes de rue égyptiens ont dû faire face pendant, et après, la révolution qui eut lieu au Caire en 2011. L'article se concentre sur l'évolution d'une telle situation, oscillant entre liberté d'expression et nouvelles formes de censure, et il se focalise sur la manière dont la censure et la peur de la censure ont façonné les contre-discours des différents artistes. Les nombreuses photographies qui illustrent le propos, toutes prises par l'auteure de l'article in situ pendant les faits, permettent de prendre la mesure de l'impact qu'ont eu ces dessins de rue en tant que nouveau medium d'expression pour des artistes anonymes à un moment difficile de l'histoire du pays.

C'est ensuite l'article de Vanessa Lee «(In)soumise à la censure : l'activisme littéraire et politique de Suzanne Roussi-Césaire » qui attire l'attention du lecteur sur la biographie et la position particulière occupée par une femme qui, bien souvent mais indûment, est restée dans l'ombre de la popularité de son époux: Suzanne Roussi-Césaire. En tant qu'épouse d'Aimé Césaire, la vie et les réalisations de cette femme n'ont pas reçu l'attention qu'elles méritaient et l'article de V. Lee nous rappelle que Suzanne RoussiCésaire était elle-même une écrivaine et militante qui créa par exemple la revue littéraire et culturelle Tropiques, et qui publia plusieurs essais importants sur la psychanalyse, l'identité caribéenne, le mouvement surréaliste, voire des réflexions sur l'exotisme. Elle fut aussi dramaturge, même si l'on peut déplorer le fait que la plupart des pièces de l'auteure ont aujourd'hui disparu. La contribution de V. Lee conduit le lecteur à percevoir en Suzanne Roussi-Césaire une épouse mais aussi et surtout une femme engagée qui mériterait d'occuper une place prééminente parmi les écrivaines caribéennes de la première moitié du vingtième siècle. 
27 Dans la seconde partie de ce recueil, l'accent sera mis sur les différentes stratégies mises en place par les artistes pour contourner ou circonvenir la censure et trouver des moyens d'expression inhabituels. Tout d'abord, l'article de Guy Lavorel, «Littératures, langues et cultures de Louisiane: faire face aux censures", explore la situation particulière à laquelle sont confrontées les littératures, les langues et les cultures de l'ancienne colonie française de Louisiane et montre comment la censure a pu être contrée dans cette vaste région. Après avoir expliqué la situation historique de la Louisiane, cet article s'intéresse à l'utilisation des langues et surtout du "cadien " ayant permis de résister aux contraintes imposées en Louisiane, ce dernier ayant perduré grâce à la création de revues et journaux et à la publication de livres. La musique s'est aussi avérée essentielle, qu'il s'agisse du jazz ou de la musique « zydeco ». L'article de Florence Labaune-Demeule («La Voix, la radio et le sermon. Censure et résistance dans The Dew Breaker d'Edwige Danticat ») présente une analyse de ce roman publié en 2004 en se concentrant plus particulièrement sur le chapitre final éponyme construit comme une nouvelle. Cet article étudie les spécificités du discours et des voix dans le contexte de la dictature des Duvalier et de la terreur engendrée par un régime qui pratiquait largement la censure. Il montre ensuite comment le trauma condamne victimes et bourreaux pour les réduire au silence, les obligeant alors à avoir recours à des moyens d'expression inhabituels, ce que s'attache à dévoiler ce roman en proposant un discours narratif de la révélation qui parvient à dépasser le silence imposé en usant paradoxalement de stratégies narratives implicites.

Dans le troisième essai de cette seconde partie, Patricia Donatien étudie deux ouvrages caribéens, l'un écrit par un auteur francophone, Roland Brival, le second par une écrivaine anglophone, Olive Senior. Cette contribution, «Autocensure et dé-censure dans Nègre de personne de Roland Brival et The Pain Tree d'olive Senior ", illustre le fait que ces deux romans constituent des espaces de liberté où des stratégies narratives particulières occupent une position centrale et permettent d'exprimer ce qui demeurerait autrement non-dit ou inaudible. Ces caractéristiques d'écriture y sont aussi présentées comme les éléments constituants d'une esthétique littéraire caribéenne.

Enfin, Karine Chevalier analyse les stratégies narratives proposées par Nabile Farès pour affronter la censure. Son article, intitulé « Nabile Farès et la censure : du silence des mots aux échos des images", s'intéresse à la manière dont l'auteur combat de nombreuses formes de censure dans l'Algérie d'après l'indépendance. Pratiquant l'autocensure, l'écrivain a recours à un style d'écriture opaque dominé par un symbolisme visuel et typographique. K. Chevalier démontre aussi que, dans ses romans graphiques plus tardifs, dont les illustrations ont été réalisées par Kamel Khélif, Farès propose une nouvelle approche d'un style dialogique où des voix polyphoniques créent des échos entre texte et images, entre auteur et dessinateur, ces derniers illustrant, par leur entreprise visuelle et textuelle, à quel point il leur paraît essentiel de vivre et de créer côte à côte, - non pas seulement d'être physiquement assis côte à côte, mais d'être unis dans une vision commune.

31 Pour conclure, un second article de Patricia Donatien, « Postcolonial et/ou (dé)colonial en France et dans la Caraïbe française, visées heuristiques et politiques: Can the Subaltern Speak?", permettra d'élargir l'approche de la censure dans les mondes coloniaux et postcoloniaux pour questionner le concept de décolonialité en étudiant les conséquences de la censure. Le concept de décolonialité pourrait-il ouvrir de nouvelles 
perspectives ? Après avoir défini les concepts de «colonial » et de " postcolonial », cet article questionne les différentes acceptions du terme « décolonial ». Selon P. Donatien, chercher le «delinking» décrit par W. Mignolo pourrait finalement ne pas s'avérer la solution requise par les chercheurs caribéens. L'auteur de l'article avance qu'aujourd'hui le rôle de ces derniers devrait être de proposer de nouvelles solutions aux problèmes générés par la colonisation et soulevées par le monde postcolonial en offrant une décolonisation des esprits, en insistant sur la nécessité de se départir de tout cadre mental lié à la colonisation, de toute idée de subalternité, et de toute demande de réparation. Elle ajoute qu'il est essentiel d'établir un vrai dialogue entre les populations anciennement colonisées et la métropole colonisatrice, position qui rappelle la théorie de la Relation développée par Édouard Glissant.

Enfin, il nous semble important de signaler que la publication de ce recueil d'articles consacré à l'expression et aux manifestations de la censure dans les productions culturelles postcoloniales a été rendue possible grâce au centre de recherches IETT (Université de Lyon, Université Jean Moulin Lyon 3, EA 8146) et à son directeur, le Professeur Gregory B. Lee. Qu'il soit remercié ici pour la confiance ainsi témoignée. Cette publication n'aurait pas non plus pu voir le jour sans l'aide de l'équipe de rédaction de la revue Transtext(e)sTranscultures, en particulier Sophie Coavoux et Gwennaël Gaffric, dont nous mesurons l'investissement.

Nous voudrions aussi adresser toute notre gratitude aux auteurs des articles, pour la richesse de leur pensée et l'intérêt qu'ils ont porté au contexte postcolonial dans toute sa diversité: Karine Chevalier, Patricia Donatien, Hajer Elarem, Charles Forsdick, Jacqueline Jondot, Guy Lavorel et Vanessa Lee.

Enfin nous souhaitons remercier ici tous ceux qui, d'une manière ou d'une autre, ont été impliqués dans l'élaboration de cette publication et dans celle des journées d'étude internationales qui l'ont précédée et dont certains textes sont présentés ici.

\section{NOTES}

1. Julien Duval, «Censure », in Universalis éducation [en ligne]. Encyclopœdia Universalis, Consulté le 7 Juin 2017, http://www.universalis-deu.com.ezscd.univ-lyon3.fr/encyclopedie/censure.

2. On se place alors du point de vue de la morale qui, pour les censeurs, ne serait pas respectée ou pourrait être menacée.

3. André Lalande, Vocabulaire technique et critique de la philosophie, Paris, PUF, coll. Quadrige, [1926], 2010, p. 133.

4. John McLeod, Beginning Postcolonialism, Manchester, Manchester University Press, 2000, p. 20-21. «La violence de cette 'révision' de son identité est véhiculée avec puissance par l'image de l'amputation. Fanon se sent amputé, violé, emprisonné par une façon de voir qui lui interdit le droit de définir sa propre identité comme sujet ; [...] Cette distinction imaginative qui permet d'établir une différence entre 'l'homme' (soi) et 'l'homme noir' (autre) constitue une part importante, dévastatrice de l'arsenal de la domination coloniale, qui emprisonne l'esprit aussi sûrement que des chaînes emprisonnent le corps ». Nous traduisons. 
5. «Parmi les censures les plus radicales, les plus sûres et les mieux cachées, il y a celles qui excluent certains individus de la communication. [...] On ne parle pas au premier venu; le premier venu ne 'prend' pas la parole. Le discours suppose un émetteur légitime s'adressant à un destinataire légitime, reconnu et reconnaissant. " (Pierre Bourdieu, «L'économie des échanges linguistiques ", In Langue française, $n^{\circ} 34$, 1977. Linguistique et sociolinguistique. p. 17-34; doi : https://doi.org/10.3406/lfr.1977.4815,

https://www.persee.fr/doc/ lfr_0023-8368_1977_num_34_1_4815, consulté le 04 mai 2018, p. 20.

6. "La disposition qui porte à "se surveiller" ", à se "corriger", à rechercher la "correction" par des corrections permanentes n'est pas autre chose que le produit de l'introjection d'une surveillance et de corrections qui inculquent sinon la connaissance, du moins la reconnaissance de la norme linguistique ; [...] En se "surveillant", les dominés reconnaissent en pratique sinon la surveillance des dominants (bien qu'ils ne « se surveillent » jamais autant qu'en leur présence), du moins la légitimité de la langue dominante.» (Bourdieu, «L'économie des échanges linguistiques", p. 26) Voir également Laurent, Martin, "Censure répressive et censure structurale : comment penser la censure dans le processus de communication?", Questions de communication, [en ligne], 15, 2009, mis en ligne le 01 août 2011. Consulté le 27/08/2020, URL : http://journals.openedition.org/questionsdecommunication/461

7. "To what extent did colonial power succeed in silencing the colonised? ", Ania Loomba, Colonialism/Poscolonialism, Second Edition, London \& New York, Routledge, The New Critical Idiom, 2005, [First Edition, 1998], p. 192. Nous traduisons.

8. "The silencing and marginalizing of the post-colonial voice by the imperial centre; the abrogation of this imperial centre within the text; and the active appropriation of the language and culture of that centre. » Bill Ashcroft, Gareth Griffiths, Helen Tiffin, The Empire Writes Back, London \& New York, Routledge, 1989, p. 83. Nous traduisons.

9. Voir Ashcroft, Griffiths, Tiffin, The Empire Writes Back, p. 84.

10. Ashcroft, Griffiths, Tiffin, The Empire Writes Back, p. 84. Nous traduisons : «Ce silence se révèle de façon spectaculaire et littérale dans la censure exercée par le gouvernement sur les journaux, les revues et une grande partie de la littérature. Il présente deux aspects : il y a l'imposition littérale du silence qui ne conférera pas la liberté nécessaire à l'appropriation de la langue, et il y a la seconde forme de silence qui précède nécessairement l'acte d'appropriation. »

11. Ashcroft, Griffiths, Tiffin, The Empire Writes Back, p. 91. Nous traduisons: «Si la langue construit le monde, alors les marges sont le centre et peuvent le reconstruire en suivant un modèle différent de conventions, d'attentes et d'expériences. "

12. "The development of independent literatures depended upon the abrogation of this constraining power [the direct control of the imperial ruling class] and the appropriation of language and writing for new and distinctive usages.» (Ashcroft, Griffiths, Tiffin, The Empire Writes Back, p. 6). «L'expansion de littératures indépendantes dépendait de l'abrogation de ce pouvoir contraignant [le pouvoir direct qu'effectuait la classe dominante de l'Empire] et de l'appropriation de la langue et de l'écriture pour en faire des usages novateurs et différents. » Nous traduisons.

13. Elleke Boehmer, Colonial \& Postcolonial Literature, Oxford \& New York, Oxford University Press, 1995, p. 3. Nous traduisons: «Plutôt que d'être simplement l'écriture qui se fit jour après l'empire, la littérature postcoloniale est une littérature qui étudie la relation coloniale d'un point de vue critique. C'est une écriture qui a pour ambition, d'une manière ou d'une autre, de s'opposer aux perspectives colonialistes. Tout comme elle requérait un changement de pouvoir, la décolonisation a exigé une révision symbolique, un remodelage des significations dominantes. La littérature postcoloniale faisait partie de ce processus de révision. »

14. Voir ce que Justin. D. Edwards appelle «the mastery of the gun et «the mastery of discourse ", Justin D. Edwards, Postcolonial Literature. A Reader's Guide to Essential Criticism, Basingstoke \& New York, Palgrave Macmillan, 2008, p. 64. 
«[...] the manipulation of language and thought [that] becomes a form of control that empowers the colonizer and subjugates the native. " Justin D. Edwards, Postcolonial Literature. A Reader's Guide to Essential Criticism, Basingstoke \& New York, Palgrave Macmillan, 2008, p.64. Nous traduisons.

15. Edwards, Postcolonial Literature, p. 66. «La violence épistémique se fait jour lorsque les personnes détentrices du pouvoir se mettent à réduire au silence un groupe moins puissant. Elle souligne ainsi l'interaction entre les structures pré-coloniales et coloniales des discours dominants qui effacent l'espace d'où s'expriment les dominés. [...] Bien souvent, le subalterne essaie de s'auto-représenter, mais cet acte de représentation n'est pas entendu. L'auditeur en position hégémonique ne le reconnaît pas, parce qu'il ne s'intègre pas aux structures institutionnelles officielles de représentation. [...] [A]u sein même de la définition de la subalternité se trouve une incapacité-à-émettre-des-actes-de-langage implicite, car si le subalterne pouvait parler, [il] ne serait plus un subalterne. » Nous traduisons.

16. J. D. Edwards parle du « dominant rhetorical mode of censorship » : « [...] the main goal of this article is to show how issues of censorship, in particular their cultural and political implications rather than their impact in terms of textual philology - are crucial to at least one definition of postcolonial literature, considering allegory as its dominant rhetorical mode (Jameson 1986, Slemon 1988). » Nous traduisons : « [...] le but principal de cet article est de montrer comment des questions liées à la censure, en particulier leurs implications culturelles et politiques plus que leur impact en termes de philologie textuelle sont essentielles à au moins une définition de la littérature postcoloniale qui considère l'allégorie comme sa stratégie rhétorique dominante (Jameson 1986, Slemon 1988). »

17. Lorenzo Mari, «'A War Between Buffoons’? Censorship and Self-Censorship in Postcolonial Literature ", Censura e autocensura, Eds. A. Bibbò, S. Ercolino, M. Lino, Between, V.9 (2015), 2, http://www.betweenjournal.it/, consulté en juin 2017. Nous traduisons : «Le lien formé par l'allégorie et l'histoire s'avère différent dans la littérature occidentale, où les allégories ont pour objet soit de 'racheter, soit d'excaver le passé', et dans la littérature postcoloniale, où les allégories visent spécifiquement à transformer 'le mythe impérial de l'histoire' (1988:158). En redonnant vie à ces histoires qui ont été systématiquement niées et/ou absentes des récits coloniaux, la littérature postcoloniale est engagée dans la lutte contre le silence imposé aux populations colonisées, y percevant une forme particulière de censure coloniale (Chin 2009). Dans le même temps, la transformation de l'histoire qui est inhérente à la littérature postcoloniale se fonde sur une série d'éléments qui apparaissent inévitablement comme 'nouveaux' et 'autres'. Cela conduit à percevoir une 'dualité paradoxale ou une certaine ambivalence' puisque la littérature postcoloniale est 'déjà constituée au sein de contraintes institutionnelles et génériques qui ont pour objet de conditionner et de déplacer la force contrediscursive [...] derrière une apparence de secondarité, de dérivation, de simulacre ou d'imitation' (Slemon 1989 :100).»

18. Rappelons ici que cette auteure sud-africaine fut elle-même victime de la censure lors de la parution de trois de ses romans: The Late Bourgeois World, A World of Strangers, Burger's Daughter. Elle établit de ce fait un lien immédiat entre son pays soumis à l'Apartheid et sa fonction d'écrivaine: "I am no stranger to censorship, living in South Africa " (Nadine Gordimer, "Censorship - The Final Solution. The Case of Salman Rushdie», 1989, Telling Times. Writing and Living, 1954-2008, New York, W.W. Norton \& Comp, Inc., 2010, p. 447-450, p. 447); « Je ne suis pas étrangère à la censure puisque je vis en Afrique du Sud. » Nous traduisons.

19. Nadine Gordimer, "Censorship - The Final Solution. The Case of Salman Rushdie", 1989, Telling Times. Writing and Living, 1954-2008, New York: W.W. Norton \& Comp, Inc., 2010, p. 447-450, p. 447. Nous traduisons : "Émeutes, autodafés, exigences d'interdiction d'un ouvrage à l'échelle mondiale, boycott des maisons d'édition, menace de renversement d'un premier ministre, cinq morts - un livre a-t-il jamais été le prétexte à un tel déferlement de sauvagerie bien-pensante? » 
20. Gordimer, “Censorship - The Final Solution", p. 348. « ...[le] principe inhérent à la censure a toujours été, est et sera toujours d'entraver le monde au char des tyrans. Le tyran peut être un dictateur, un régime politique, ou une bigoterie morale ou religieuse. » Nous traduisons.

21. Gordimer, "Censorship - The Final Solution", p. 348.

22. "From the corpus of language, within that guilt shared with fellow writers, the writer fashions his entreprise, which then becomes his 'essential gesture as a social being'. (Nadine Gordimer, "The Essential Gesture", 1985, Telling Times. Writing and Living, 1954-2008, New York: W.W. Norton \& Comp, Inc., 2010, p. 409-424, p. 410.) Nous traduisons : «À partir du corpus de la langue et avec la culpabilité qu'il partage avec ses homologues, l'écrivain façonne son entreprise créative qui devient alors son « acte essentiel en tant qu'être social. »

23. Voir Gordimer, "The Essential Gesture", p. 410.

24. Voir Gordimer, "The Essential Gesture”, p. 417-418.

25. Gordimer, "The Essential Gesture”, p.420. Nous traduisons : "Qu'un écrivain soit noir ou blanc, en Afrique du Sud, l'acte essentiel par lequel il entre dans une relation de fraternité avec les hommes - ce qui est la seule définition de la société qui ait une validité permanente - est un acte révolutionnaire. »

26. Nous traduisons : «Les écrivains qui acceptent une responsabilité professionnelle dans la transformation de la société cherchent toujours des manières de le faire que leur société ne pourrait jamais imaginer, et encore moins exiger: se contraindre à trouver des moyens qui s'enfonceront, tels un foret, pour faire surgir le premier jaillissement de créativité, pour en inonder les censeurs, nettoyer la législation de la pornographie de leurs lois racistes et sexistes, laver au jet les différences religieuses, éteindre les bombes au napalm et les lance-flammes, dépolluer la terre, la mer et les airs et permettre aux êtres humains d'accéder à la source estivale et éphémère de la joie pure. On pourrait aussi reconnaitre qu'il s'agit là de tout ce que les écrivains peuvent faire : car la créativité vient de l'intérieur, elle ne peut être le résultat d'un acte de volonté ni être dictée si elle ne s'y trouve pas déjà, bien qu'elle puisse être anéantie sur ordre. La transformation de l'expérience demeure l'acte essentiel et fondamental de l'écrivain, l'apparition de quelque chose de limité qui ne révèle sa vraie signification que lorsque l'imagination de l'écrivain a pu lui donner toute son ampleur. » Elle ajoute : « L'écrivain est à jamais à la recherche de l'entéléchie dans sa relation avec la société qui est la sienne. Partout dans le monde, il doit avoir la possibilité d'être seul et en même temps d'entretenir une relation vitale avec les autres ; il a besoin d'une liberté artistique et sait qu'elle ne peut exister hors de son contexte étendu ; il perçoit les deux présences qui sont en lui - un égocentrisme créatif et une juste conscience - et il doit essayer de savoir s'ils sont liés dans une lutte à mort ou s'ils sont en fait des fœetus partageant une relation gémellaire féconde. » (Notre traduction pour : « The writer is eternally in search of entelechy in his relation to his society. Everywhere in the world, he needs to be left alone and at the same time to have a vital connection with others; he needs artistic freedom and knows it cannot exist without its wider context; feels two presences within - creative selfabsorption and conscionable awareness - and must resolve whether these are locked in death struggle, or are really foetuses in a twinship of fecundity. ", Gordimer, "The Essential Gesture", p. 422 \& p. 424).

27. Gordimer, “The Essential Gesture”, p. 424.

28. Nadine Gordimer, "Censorship and its Aftermath", Telling Times. Writing and Living, 1954-2008, New York, W.W. Norton \& Comp, Inc., 2010, p. 463-472.

"Political censorship has taken place of first importance since before the second half of our century. And it has been taken over, surely as never before, by the knuckle-duster imprisonment of writers and journalists, the banning of individual writers, the closure of newspapers, the prosecution of editors, the exclusion of television crews and journalists from the scenes of events - all under laws that make conventional censorship appear namby-pamby. Repressive regimes [...] have maintained themselves with these laws that, at first appearing ancillary to censorship, 
ended by rendering it old hat, almost redundant. » (Gordimer, "Censorship and its Aftermath" ", p. 463) Nous traduisons : "La censure politique occupe une place de première importance depuis bien avant la seconde moitié de ce siècle. Et elle s'est manifestée, sans aucun doute comme jamais auparavant, par l'emprisonnement coup de poing d'écrivains et de journalistes, le bannissement d'auteurs individuels, la fermeture de journaux, les poursuites judiciaires à l'encontre des rédacteurs, l'éviction d'équipes de télévision et de journalistes de certains théâtres d'opérations tout cela sous couvert de lois qui donnent à la censure conventionnelle des airs débonnaires. Les régimes répressifs [...] ont réussi à se maintenir grâce à ces lois qui, sous des apparences d'abord complémentaires à la censure, ont fini par lui donner des apparences vieux jeu, presque obsolètes. "

Nous traduisons : « [They get] used to it, which means that they tried to [defy] censorship and/or found ways round it. » (Gordimer, "Censorship and its Aftermath", p. 469).

29. "While we rejoice at new freedom for writers in many countries long denied it, and work for freedom for writers in those countries where the many devices of censorship still prevail [...], we must also remember that writers are never freed of the past. Censorship is never over for those who have experienced it. It is a brand on the imagination that affects the individual who has suffered it, for ever.» (Gordimer, 1990, 471) Nous traduisons : " Alors que nous nous réjouissons de voir que dans de nombreux pays, des écrivains qui en avaient longtemps été privés ont pu bénéficier d'une liberté retrouvée et alors que nous travaillons pour obtenir plus de liberté pour les écrivains de ces pays où de nombreuses mesures de censure perdurent toujours, [...] nous devons aussi nous souvenir que les écrivains ne sont jamais libérés du passé. La censure n'est jamais révolue pour ceux qui y ont été soumis. C'est un fer apposé sur l'imagination qui affecte l'individu qui en a souffert, et cela pour toujours.»

30. Nous traduisons : «the well-earned role of writer-as-writer in the post-colonial era. " (Nadine Gordimer, «The Status of the Writer in the World Today » (1997), Telling Times. Writing and Living, 1954-2008, New York, W.W. Norton \& Comp, Inc., 2010, p. 520-531, p. 525)

31. Voir Charles Forsdick, «Between Censorship and Amnesia: The End of the Penal Colony in French Guiana », page finale.

\section{AUTEUR}

\section{FLORENCE LABAUNE-DEMEULE}

Florence Labaune-Demeule est Agrégée et Professeur des Universités en anglais et en littératures postcoloniales à l'Université Jean Moulin Lyon 3, Lyon, France. Plus particulièrement spécialisée dans les littératures postcoloniales caribéennes et indiennes, elle a publié de nombreux articles sur des auteurs comme V.S. Naipaul, Derek Walcott, Jean Rhys, Fred D'Aguiar, Edwidge Danticat, Jamaica Kincaid, ou Anita Desai, Anita Nair, Arundhati Roy, etc. Elle a également publié des ouvrages, dont une monographie consacrée à The Enigma of Arrival de V.S. Naipaul (V.S. Naipaul. L'Énigme de l'arrivée. L'éducation d'un point de vue, 2007), et plusieurs recueils d'articles.

Florence Labaune-Demeule is Professor in English and Postcolonial Literatures at Jean Moulin Lyon 3 University, Lyon, France. Her research interests focus on Anglophone Caribbean and Indian literatures. She has written extensively on writers of Caribbean or Indian descent like V.S. Naipaul, Derek Walcott, Jean Rhys, Fred D’Aguiar, Edwidge Danticat, Jamaica Kincaid, Anita Desai, 
Anita Nair, Arundhati Roy, etc. She has also published a few books, among which a monograph about V.S. Naipaul's The Enigma of Arrival (V.S. Naipaul. L'Énigme de l'arrivée. L'éducation d'un point de vue, 2007) and a few collections of essays. 